\title{
Commissioning of a photoelectron spectrometer for soft X-ray photon diagnostics at European XFEL
}

\author{
Joakim Laksman, $^{a *}$ Jens Buck, ${ }^{b, c}$ Leif Glaser, ${ }^{b}$ Marc Planas, ${ }^{a}$ \\ Florian Dietrich, ${ }^{a}$ Jia Liu, ${ }^{a}$ Theophilos Maltezopoulos, ${ }^{a}$ \\ Frank Scholz, ${ }^{b}$ Jörn Seltmann, ${ }^{b}$ Gregor Hartmann, ${ }^{b, d}$ Markus Ilchen, ${ }^{a, d}$ \\ Wolfgang Freund, ${ }^{a}$ Naresh Kujala, ${ }^{a}$ Jens Viefhaus ${ }^{b, e}$ And Jan Grünert ${ }^{a}$ \\ ${ }^{a}$ European XFEL, Holzkoppel 4, 22869 Schenefeld, Germany, ${ }^{b}$ Deutsches \\ Elektronen-Synchrotron DESY, Notkestraße 85, 22607 Hamburg, Germany, \\ ${ }^{c}$ Christian-Albrechts-Universität zu Kiel, Institut für Experimentelle und Angewandte \\ Physik, Leibnizstraße 19, 24098 Kiel, Germany, ${ }^{d}$ Institut für Physik, University of \\ Kassel, Heinrich-Plett-Straße 40, 34132, Kassel, Germany, and ${ }^{e}$ Helmholtz-Zentrum \\ Berlin für Materialien und Energie, Berlin, Germany. \\ E-mail: joakim.laksman@xfel.eu
}

Photon diagnostics; Photoelectron Spectrometer; Polarimeter; EuXFEL; FEL

\begin{abstract}
We are describing the commissioning and first operation of an angle resolved photoelectron spectrometer for non-invasive shot-to-shot diagnostics at the European XFEL soft X-ray beamline. Our objective with the instrument is to provide the users and operators with reliable pulse resolved information regarding photon energy and polarization that opens up a variety of applications for novel experiments but also hardware optimization.
\end{abstract}




\section{Introduction}

The principle of linear accelerator based Self Amplification of Spontaneous Emission (SASE) is that a collimated electron bunch is accelerated to almost the speed of light prior to injection in a periodic magnetic field undulator where electromagnetic radiation is generated. Spatial modulation of electron bunches takes place as they interact with the electro-magnetic field leading them to ultimately radiate in phase and producing highly coherent and intense X-ray pulses (Pellegrini et al., 2016). The unique properties of X-ray Free-Electron Laser (XFEL) radiation with high intensity (up to $\sim 10^{12}$ photons/pulse), high repetition rate and short pulse length ( 1 fs to $100 \mathrm{fs}$ ) has found applications in physics, chemistry, biology and medicine where the ability to take snapshots of samples on a molecular length and time scale provides a wealth of new information. The construction of XFEL facilities around the world has created a need for instrumentation development for diagnostics and data acquisition that is capable of handling these requirements without damage or being overloaded but at the same time provide relevant beam parameters (Ullrich et al., 2012; Feldhaus et al., 2013; Yabashi et al., 2013; Bostedt et al., 2016; Seddon et al., 2017; Kraus et al., 2018).

At the European XFEL (EuXFEL) facility in Schenefeld, Germany, the X-ray photon diagnostics group (XPD) utilize a large array of techniques for providing beam parameters to users and operators (Grünert et al., 2019; Tschentscher et al., 2017). A non-invasive principle for photon diagnostics is the usage of low density matter where gas targets are ionized by XFEL pulses and only the resulting ions or electrons are detected (Braune et al., 2016). Two complementary gas based techniques that provide pulse resolved information are (1) X-ray Gas Monitor (XGM) that gives beam position and absolute pulse energy (Tiedtke et al., 2009; Maltezopoulos et al., 2019) and (2) Photoelectron Spectrometer (PES) that gives spectral distribution and polarization.

In this paper we describe the PES instrument for diagnostics installed in the SASE3

IUCr macros version 2.1.10: 2016/01/28 
beamline. It is located in tunnel XTD10 (3142 $\mathrm{m}$ from the injector) downstream of the XGM and upstream of the gas-attenuator (GATT), horizontal offset mirrors (HOMS) and the monochromator. The location is chosen to provide better feedback for FEL control to know in which direction the photon energy deviates in shots where it is not matching the monochromator. The beamline with variable-gap undulators can provide soft X-rays in the range $250 \mathrm{eV}$ to $3000 \mathrm{eV}$ to the scientific endstations Small Quantum Systems (SQS) and Spectroscopy and Coherent Scattering (SCS). At the moment only horizontal polarization is possible, but with the future installation of an afterburner (Lutman et al., 2016), circular and elliptical polarization will be provided to the users. SASE is a stochastic process where pulses have individual characteristics. The purpose of this instrument is, accordingly, to provide machine operators and users shotto-shot information about photon energy spectrum and the polarization. This has a wide range of applications: Experiments at resonances where narrow and precise bandwidth is crucial will use the monochromator to select their desired photon energy, but they will rely on precise undulator tuning which can only be achieved with the noninvasive diagnostics that the PES provides (Braune et al., 2018). Studies of magnetic material properties and circular dichroism will require full polarization control and monitoring of the Stokes parameters. Objectives for the PES instrument are defined by the user requirements which are relative photon energy resolution $10^{-3}$ to $10^{-4}$ and determination of the X-ray polarization vector with $1 \%$ accuracy. These values must be supplied to operators for efficient tuning of the undulator gap and the future afterburner. In addition, they must be collected and stored in the same data file as the user experiment for cross correlation and data sorting. An additional application of the PES that will be implemented in SASE3 is to send the evaluated center wavelength to the XGM DOOCS server for cross checking with the value taken from the undulator server. Furthermore due to data transfer capacity limitations, 2D pixelated imaging 
detectors at the end stations can currently process and store data from only up to 800 of the 2700 pulses that are potentially available from the machine within one pulse train. To optimize the amount of useful data, a veto system is being implemented at EuXFEL. Whenever the PES encounters a pulse with undesired photon energy and/or polarization, a signal is sent to the imaging detector, prompting it to overwrite the data of this recorded pulse with later pulses from the same pulse train. With this routine, we ensure that as much as possible of the storage capacity indeed contain high quality data.

\section{Instrument}

Electron spectrometers based on the Time-of-flight (TOF) concept have drifttubes with tunable voltages that decelerate the electrons. Fast electronics register the time difference from ionization to detection which is related to the kinetic energy of the photoelectrons. The PES presented here, is based on a design made originally for the online diagnostic unit of beamline P04 at PETRA III, DESY, Hamburg, Germany (Viefhaus et al., 2013) which has previously been operated at several FEL facilities and proven itself as a versatile tool for photon diagnostics (Allaria et al., 2014; Ferrari et al., 2015; Hartmann et al., 2016; Roussel et al., 2017; von Korff Schmising et al., 2017), angular streaking (Düsterer et al., 2016; Hartmann et al., 2018), and multi-photon physics (Ilchen et al., 2012; Ilchen et al., 2018). To achieve angular photoemission resolution with respect to the polar angle, the PES consists of sixteen electron TOF spectrometers oriented perpendicular to the X-ray beam, see Fig. 1. The drifttubes

of the EuXFEL PES are labeled $\left[\begin{array}{llllll}N & N N E & N E & E N E & E & \ldots\end{array}\right]$, where $N$ and $S$ are positioned vertical while $E$ and $W$ are positioned horizontal, left and right respectively seen from upstream. An advantage of having sixteen spectrometers is the high redundancy that results e.g. for low energy pulses or photon energy regions with 
low cross-section. More reliable spectral data can be retrieved by summing different channels together achieving higher single pulse statistics.

In the case of the EuXFEL PES a target gas which can be a rare gas ( $\mathrm{Ne}, \mathrm{Kr}$ or $\mathrm{Xe}$ ) or $\mathrm{N}_{2}$ is injected as an effusive jet in the interaction region center via a $\varnothing 100 \mu \mathrm{m}$ inner diameter capillary. Reference data for the gas targets can be found for crosssections (Yeh \& Lindau, 1985), anisotropy parameter (Derevianko et al., 1999; Lindle et al., 1984) binding energy (Cardona \& Ley, 1978) and linewidth (Prince et al., 1999). The four gas targets available for the PES inside the EuXFEL tunnel are selected with the motivation of covering the entire soft X-ray region $250 \mathrm{eV}$ to $3000 \mathrm{eV}$ at SASE3. The criteria are: (1) Sufficient cross-section for single shot diagnostics. (2) Low kinetic energy of the created photoelectrons to achieve good photon energy resolution. (3) Anisotropy parameter $\beta$ far from 0 to retrieve reliable polarization. (4) Avoid overlap with Auger lines that would make the data unreliable. For example around $900 \mathrm{eV}$, the best choice is Ne 1s (see table 1) with a binding energy of $870.2 \mathrm{eV}$. At this photon energy, the cross-section is $\sigma>0.2 \mathrm{Mb}, \beta=2.00$ and the $K L L$ Auger lines do not overlap with the photoline. The base pressure in the chamber is $1 \times 10^{-8}$ mbar. Depending on the ionization cross-section we inject gas to a background pressure of $1 \times 10^{-7}$ mbar to $1 \times 10^{-5}$ mbar. An X-ray pulse ionizes the target and photo electrons are ejected anisotropically whereof a small fraction are registered by the detectors from which linear momentum data can be deduced.

The drifttubes are each composed of 4 conical electrode segments constructed of copper beryllium where we apply voltages to decelerate the electrons. The segments are labeled [A B C D] in Fig. 1. The total distance from the central symmetry interaction point to the detector is $135 \mathrm{~mm}$. The entrance hole of a drifttube is $\varnothing 3 \mathrm{~mm}$. The acceptance angle for each spectrometer is $0.2 \%$ of $4 \pi$. The PES is located far from any focus and the beam spot size is estimated to be about $\varnothing 5 \mathrm{~mm}$. Deceleration voltages are se-

IUCr macros version 2.1.10: 2016/01/28 
lected for optimizing TOF resolution and focussing electrons to the detector for optimal transmission. In general we operate with the settings $R \times\left[\begin{array}{llll}0.00 & 0.65 & 0.90 & 1.00\end{array}\right]$, where the lowest voltage is closest to the interaction region and the highest voltage is closest to the detector. The deceleration settings can be described with the retardation value $R$. We have empirically and from simulations found this potential gradient to optimize the performance. In general the kinetic energy must be reduced to about $15 \mathrm{eV}$ to $60 \mathrm{eV}$ in order to achieve sufficient resolution (Buck, 2012). Each drift-tube is enclosed with grounded aluminum sheet metal for electromagnetic shielding from the surrounding environment. Detectors are Z-stack MCPs (Hamamatsu F1552-011) with an in-house designed assembly with anodes and electronics made by the P04 group at DESY, Hamburg. A grid mesh is placed in front of the MCP stack with the same voltage as the last segment for creating a post-acceleration region near the detector to increase detection efficiency.

Data acquisition is performed with four digitizer boards (SP Devices ADQ 412-4G) in a $\mu$ TCA crate that we operate in four channel mode with $2 \mathrm{GS} / \mathrm{s}$ (500 ps binning). An option is to bridge two channels in order to increase the sample rate to $4 \mathrm{GS} / \mathrm{s}$ (250 ps binning). Their dynamic range is $\pm 400 \mathrm{mV}$. Prior to digitizers we use pre-amplifiers (Mini-circuits ZFL-1000LN+) that enhance the MCP-detector signal and also protect the expensive digitizer channels from possible overvoltages exceeding their maximum allowed value of $\pm 1 \mathrm{~V}$. For additional signal matching, the signal chain contains $3 \mathrm{~dB}$ attenuators at the input of the pre-amplifiers and $10 \mathrm{~dB}$ attenuators at the input of the digitizers. A 3D-Helmholtz-coil-structure encloses the device and is used to cancel out the background magnetic field which otherwise would influence the electron trajectories. A fluxgate type magnetometer (Bartington Mag-03) is mounted to the vacuum chamber to monitor the magnetic field. The required voltages for PES electrodes, MCP-detectors, pre-amplifiers and Helmholtz-coils are applied with a modular 
high voltage supply (MPOD). For alignment of the instrument to the beam the setup has decoupled translation and rotation motion in all six degrees of freedom controlled by stepper motors. Six fiducials are placed on the chamber to enable pre-alignment by a laser tracker. Stepper motors, magnetometer, pumps, gatevalves and pressure gauges are connected via a crate with Beckhoff modules for power and readout. MPOD, $\mu \mathrm{TCA}$ and Beckhoff crates are remotely operated via the Karabo control system which is a SCADA environment designed for both hardware control and data acquisition (Hauf, 2019).

Fig. 2 presents a functional scheme of the control and data acquisition system. The plotted voltage signal shows a typical spectrum where the first peak is the prompt from scattered light and the following peaks result from photoelectrons. The time difference gives us the TOF if we add the $0.45 \mathrm{~ns}$ it takes for the light propagation from the interaction region to the detector. In general the external trigger given from the bunch pattern is stable within the time-frame of an experiment so no new readout of the prompt is required to define a reference TOF.

\section{Results}

\subsection{Spectral distribution}

The photoelectron's kinetic energy $E_{K}$ can be written as

$$
E_{K}=\frac{\mathrm{m}_{\mathrm{e}} L^{2}}{2 T^{2}}
$$

where $m_{e}$ is electron mass, $L$ is the distance the electrons travel and $T$ is the TOF. Indeed, for $0 \mathrm{~V}$ retardation where no electric field penetration is present, the electron TOF spectra in principle contains sufficient information for an intrinsic absolute photon energy calibration if one peak can be identified. Since we have $L=135 \pm 1 \mathrm{~mm}$ and $T$ is measured, the value $E_{K}$ can be calculated. Fig. 3 (a) presents a TOF spectrum for Ne 1s

with binding energy $E_{B}=870.2 \mathrm{eV}$ and natural linewidth $0.24 \mathrm{eV}$ collected at photon IUCr macros version 2.1.10: 2016/01/28 
energy $h \nu=914 \mathrm{eV}$. Data is summed over 100 pulses to improve statistics. The photon energy calibration of the SASE3 undulator gap has been done over the entire photon energy range with the K-monochromator (Freund et al., 2019). At this photon energy, the cross-section is $\sigma=0.2 \mathrm{Mb}$ and the anisotropy parameter is $\beta=2.00$. Peak center TOF is $34.25 \mp 0.2 \mathrm{~ns}$ which from Eq. 1 leads to $E_{K}=44.2 \pm 1.1 \mathrm{eV}$. Adding the kinetic energy with the binding energy gives the photon energy, $\overbrace{44.2 \mathrm{eV}}^{E_{K}}+\overbrace{870.2 \mathrm{eV}}^{E_{B}} \approx \overbrace{914 \mathrm{eV}}^{h \nu}$, thus confirming that the undulator gap calibration is correct. In Fig. 3 (a), the red curve shows kinetic energy as a function of TOF from Eq. 1. Furthermore, from the Ne 1s peak in Fig. 3 (a) we estimate a spectral bandwidth of $\sim 0.9 \%$ which is in excellent agreement with the bandwidth that we find from scanning over the monochromator and registering the intensity on an imager.

Fig. 3 (b) presents TOF spectra at detector $E$ for $\mathrm{N}_{2} 1$ s with $E_{B}=409.9 \mathrm{eV}$ and natural linewidth $0.115 \mathrm{eV}$ at photon energies $899 \mathrm{eV}, 904 \mathrm{eV}$ and $914 \mathrm{eV}$. Data was also collected for many more photon energies up to $1000 \mathrm{eV}$. At these photon energies the cross-section is $\sigma=0.09 \mathrm{Mb}$ and the anisotropy parameter is $\beta=2.00$. The retardation voltage was kept constant at $-480 \mathrm{~V}$. To provide pulse resolved spectra, a mapping function must be constructed that converts TOF to kinetic energy for the applied retardation value $R$. In the situation of $R \neq 0$ due to field penetration in the drift-tubes, instead of Eq. 1 we derive a generalized expression

$$
E_{K}=P_{R}+\frac{P_{L}}{\left(T+P_{T}\right)^{2}}
$$

where $P_{R}$ is a parameter that is related to the retardation voltage, $P_{L}$ is related to drifttube length and $P_{T}$ is related to shift in TOF. The three parameters are characteristic for a certain value $R$ and can be used to calculate $E_{K}$ regardless of photon energy and target gas. Fig. 3 (b) plots kinetic energy as a function of the TOF spectral peak mean value (black dots) for channel E. Eq. 2 has been fitted to the data (red curve). The sixteen individual drifttubes have similar but non-identical parameters $P_{R}, P_{L}$ and IUCr macros version 2.1.10: 2016/01/28 
$P_{T}$ which must be calculated for each of them. Fig. 3 (b) furthermore demonstrates the PES energy resolution performance. The spectra at $899 \mathrm{eV}$ and $904 \mathrm{eV}$ overlap but have clearly distinguished mean-values. We estimate that an energy difference of $1 \mathrm{eV}$ can be separated. Therefore at this kinetic energy $(\sim 500 \mathrm{eV})$, neglecting the contribution of the SASE FEL bandwidth, we achieve a resolution of $\sim 2 \times 10^{-3}$ which corresponds to $\sim 1 \times 10^{-3}$ photon energy resolution.

\subsection{Polarimetry}

Normalization of the 16 detector signals is accomplished with Auger lines whose intensity distribution is nearly isotropic in both polar and azimuth directions (Cleff \& Mehlhorn, 1974). Auger lines can be isolated from the photoelectron lines by selecting suitable photon energy and drift-tube retardation. In particular the $\mathrm{N}_{2} K V V$ transition energies in the range $357 \mathrm{eV}$ to $367 \mathrm{eV}$ (Cryan et al., 2012) are useful for this purpose. Fig. 4 presents electron TOF data from $\mathrm{N}_{2}$ target with $867 \mathrm{eV}$ photon energy and $-340 \mathrm{~V}$ retardation voltage on eight of the channels. The data is summed over ten pulses for reliable statistics. The $K V V$ peak is present at all channels with approximately constant intensity. The $\mathrm{N}_{2}$ 1s peak intensity on the other hand shows strong angular dependence which is expected due to the horizontal polarization and anisotropical distribution of this line $(\beta=2.00)$ at this photon energy. The Peak identification has been confirmed by tuning the undulator-gap and observing that the photo line is shifting proportionally to the photon energy while the $K V V$ Auger band remains stationary. For background subtraction, we collected reference data files without gas injection but otherwise identical experimental conditions. After subtracting background we integrated the intensity of the $K V V$ band on all 16 detectors and used these values for normalizing the individual detectors to correct for their non-identical performance.

IUCr macros version 2.1.10: 2016/01/28 
We collected data for Ne $1 \mathrm{~s}$ at $914 \mathrm{eV}$. Drift-tube voltages were set to $0 \mathrm{~V}$. Fig. 5 presents intensity normalized TOF spectra from all 16 channels for a single photon pulse. First peak (red dashed) indicates scattered light which defines the start time. Photoelectron lines $2 \mathrm{~s}$ and $2 \mathrm{p}$ and also $K L L$ Auger line (black dashed) have kinetic energies from $860 \mathrm{eV}$ to $900 \mathrm{eV}$ and a TOF around $20 \mathrm{~ns}$ which cannot be resolved with this retardation voltage. The Ne 1s photoelectron is highlighted in the gray region. We see that the relative signal intensity is highest in the horizontal plane $E$ and $W$ and close to zero in the vertical direction $N$ and $S$. This indicates that the strongest component in the Stokes vector is the horizontal polarization with essentially no contribution from the vertical and also no unpolarized or circularly polarized light. When we study the intensity in all 16 detectors we get a picture of the photoelectrons angular distribution.

The red crosses in the polar plot in Fig. 6 (a) represent the peak integrals over Ne 1s from one pulse after background subtraction. We have fitted to the data a polarization function $P$ which describes the intensity as a function of the polar angle $\theta$ (Allaria et al., 2014),

$$
P(\theta)=1+\frac{\beta}{4}\left(1+3 P_{\text {Lin }} \cos (2(\theta-\psi))\right)
$$

$\beta$ is the anisotropy parameter, $P_{\text {Lin }}$ is the linear polarization component and $\psi$ is the tilt angle. For perfect horizontal polarization, we expect $P_{\text {Lin }}=1$ and $\psi=0$. For this particular pulse we retrieved the values $P_{\text {Lin }}=0.96 \pm 0.03$ and $\psi=-0.02 \pm 0.03$. Error has been estimated as a $95 \%$ confidence interval of the fitting. The symbol $\vec{\varepsilon}$ indicates the polarization vector of X-rays. Note that this pulse resolved technique is only capable of determining the linear polarization, in contrast to invasive multilayer based polarimetery (Grizolli et al., 2016) where the complete Stokes vector is found. However, for FELs, it is reasonable to assume only a negligible unpolarized contribution and that any deviation from linear polarization must be circular, either right hand or IUCr macros version 2.1.10: 2016/01/28 
left hand polarized. Similar data set as presented in Fig. 6 (a) was collected during single bunch mode for a large amount of pulse trains. Fig. 6 (b) presents $P_{\text {Lin }}$ and $\psi$ for 450 consecutive pulses, which characterizes the stability of the machine parameters.

\subsection{Veto signal}

Diagnostics-based generation of veto signals will significantly enhance the overall efficiency of the large pixelated imaging detectors at the scientific end stations, because it allows for real time selection of pulse resolved data. The PES in combination with the XGM will provide valuable input to the veto system.

In Fig. 6(b), sharp spikes can be seen for some pulses with values dramatically different from the expected interval. This data, that is usually related to too low pulse energy informs us that this pulse has non-desired characteristics. This information could be fed into the Veto-system, preventing experiments from collecting data for this particular pulse.

\section{Summary and Outlook}

We have installed the PES device in the SASE3 tunnel and integrated it in the EuXFEL control and data acquisition system. We have written algorithms that calculates spectral distribution and polarization from TOF spectra for soft X-ray photon diagnostics applications. The challenges that remain to be implemented are automatization with regard to voltage settings and gas selection in order for the device to operate unattended with minimal involvement from the users. Photon energy and polarization values must be provided in real time for both diagnostics and the Veto-system.

At present we are working on modifying the PES concept for the hard X-ray beamlines which imposes some additional challenges on the design criteria: For hard X-rays in the range $5 \mathrm{keV}$ to $25 \mathrm{keV}$, resonances for the available gas targets are sparse (see

IUCr macros version 2.1.10: 2016/01/28 
table 1) thus demanding the device to resolve high kinetic energy photoelectrons which requires longer drifttubes and higher retardation voltages. In addition, cross sections are lower which we aim to compensate with larger detectors and a synchronized pulsed gas-injection capillary which leads to higher pressure in the interaction region at the time of the ionization.

\section{Acknowledgements}

Several groups have contributed to enable operation of the PES: The P04 group at PETRA III, DESY, Hamburg, Germany, constructed the MCP-based detectors and were heavily involved in development, design and fabrication of of the drifttubes. The commissioning was performed by XPD in close collaboration with several scientific support groups from EuXFEL and DESY: X-Ray Optics (XRO), Vacuum (VAC), Advanced Electronics (AE), Information Technology and Data Management (ITDM), Control and Analysis Software (CAS), Survey and Alignment team and Transport group MEA have all provided valuable support.

\section{References}

Allaria, E., Diviacco, B., Callegari, C., Finetti, P., Mahieu, B., Viefhaus, J., Zangrando, M., De Ninno, G., Lambert, G., Ferrari, E., Buck, J., Ilchen, M., Vodungbo, B., Mahne, N., Svetina, C., Spezzani, C., Di Mitri, S., Penco, G., Trovó, M., Fawley, W. M., Rebernik, P. R., Gauthier, D., Grazioli, C., Coreno, M., Ressel, B., Kivimäki, A., Mazza, T., Glaser, L., Scholz, F., Seltmann, J., Geßler, P., Grünert, J., De Fanis, A., Meyer, M., Knie, A., Moeller, S. P., Raimondi, L., Capotondi, F., Pedersoli, E., Plekan, O., Danailov, M. B., Demidovich, A., Nikolov, I., Abrami, A., Gautier, J., Lüning, J., Zeitoun, P. \& Giannessi, L. (2014). Phys. Rev. X, 4, 041040.

Bostedt, C., Boutet, S., Fritz, D. M., Huang, Z., Lee, H. J., Lemke, H. T., Robert, A., Schlotter, W. F., Turner, J. J. \& Williams, G. J. (2016). Reviews of Modern Physics, 88(1).

Braune, M., Brenner, G., Dziarzhytski, S., Juranić, P., Sorokin, A. \& Tiedtke, K. (2016). Journal of Synchrotron Radiation, 23, 10-20.

Braune, M., Buck, J., Kuhlmann, M., Grunewald, S., Düsterer, S., Viefhaus, J. \& Tiedtke, K. (2018). Journal of Synchrotron Radiation, 25(1), 3-15.

Buck, J. (2012). Conceptual Design Report: Online Time-of-Flight Photoemission Spectrometer for X-Ray Photon Diagnostics (XFEL.EU TR-2012-002). Tech. rep. European XFEL $\mathrm{GmbH}$.

Cardona, M. \& Ley, L. (1978). Photoemission in Solids I: General Principles. Springer-Verlag, Berlin.

Cleff, B. \& Mehlhorn, W. (1974). Journal of Physics B: Atomic and Molecular Physics, 7(5), 593.

Cryan, P., Glownia, M., Andreasson, J., Belkacem, A., Berrah, N., Blaga, I., Bostedt, C., Bozek, J., Cherepkov, A., Dimauro, F., Fang, L., Gessner, O., Gühr, M., Hajdu, J., Hertlein, P., Hoener, M., Kornilov, O., Marangos, P., March, M., McFarland, K., Merdji, H., Messerschmidt, M., Petrovi, S., Raman, C., Ray, D., Reis, A., Semenov, K., Trigo, M., White, L., White, W., Young, L., Bucksbaum, H. \& Coffee, N. (2012). Journal of Physics B: Atomic, Molecular and Optical Physics, 45(5).

Derevianko, A., Johnson, W. R. \& Cheng, K. T. (1999). At. Data Nucl. Data Tables, 73(2), 153-211.

Düsterer, S., Hartmann, G., Babies, F., Beckmann, A., Brenner, G., Buck, J., Costello, J., Dammann, L., Fanis, A. D., Geßler, P., Glaser, L., Ilchen, M., Johnsson, P., Kazansky, A. K., Kelly, T. J., Mazza, T., Meyer, M., Nosik, V. L., Sazhina, I. P., Scholz, F., Seltmann, J., Sotoudi, H., Viefhaus, J. \& Kabachnik, N. M. (2016). Journal of Physics B: Atomic, Molecular and Optical Physics, 49(16), 165003.

IUCr macros version 2.1.10: 2016/01/28 
Feldhaus, J., Krikunova, M., Meyer, M., Möller, T., Moshammer, R., Rudenko, A., Tschentscher, T. \& Ullrich, J. (2013). Journal of Physics B: Atomic, Molecular and Optical Physics, 46(16).

Ferrari, E., Allaria, E., Buck, J., De Ninno, G., Diviacco, B., Gauthier, D., Giannessi, L., Glaser, L., Huang, Z., Ilchen, M., Lambert, G., Lutman, A. A., Mahieu, B., Penco, G., Spezzani, C. \& Viefhaus, J. (2015). Scientific Reports, 5(August), 1-8.

Freund, W., Fröhlich, L., Karabekyan, S., Koch, A., Liu, J., Nölle, D., Wilgen, J. \& Grünert, J. (2019). Journal of Synchrotron Radiation, ThisVolume.

Grizolli, W., Laksman, J., Hennies, F., Jensen, B. N., Nyholm, R. \& Sankari, R. (2016). Review of Scientific Instruments, 87(2).

Grünert, J., Planas Carbonell, M., Dietrich, F., Falk, T., Freund, W., Koch, A., Kujala, N., Laksman, J., Liu, J., Maltezopoulos, T., Tiedtke, K., Jastrow, U. F., Sorokin, A., Syresin, E., Grebentsov, A. \& Brovko, O. (2019). Submitted to Journal of Synchrotron Radiation.

Hartmann, G., Lindahl, A. O., Knie, A., Hartmann, N., Lutman, A. A., Macarthur, J. P., Shevchuk, I., Buck, J., Glownia, J. M., Helml, W., Huang, Z., Kabachnik, N. M., Kazansky, A. K., Liu, J., Marinelli, A., Mazza, T., Nuhn, H., Walter, P., Viefhaus, J., Meyer, M., Moeller, S., Coffee, R. N. \& Ilchen, M. (2016). Review of Scientific Instruments, $\mathbf{8 7}(2016), 083113$.

Hartmann, N., Hartmann, G., Heider, R., Wagner, M. S., Ilchen, M., Buck, J., Lindahl, A. O., Benko, C., Grünert, J., Krzywinski, J., Liu, J., Lutman, A. A., Marinelli, A., Maxwell, T., Miahnahri, A. A., Moeller, S. P., Planas, M., Robinson, J., Kazansky, A. K., Kabachnik, N. M., Viefhaus, J., Feurer, T., Kienberger, R., Coffee, R. N. \& Helml, W. (2018). Nature Photonics, 12(4), 215-220.

Hauf, S. (2019). Submitted to Journal of Synchrotron Radiation.

Ilchen, M., Deinert, S., Glaser, L., Scholz, F., Seltmann, J., Walter, P. \& Viefhaus, J. (2012). Journal of Physics B: Atomic, Molecular and Optical Physics, 45(22), 225102.

Ilchen, M., Hartmann, G., Gryzlova, E. V., Achner, A., Allaria, E., Beckmann, A., Braune, M., Buck, J., Callegari, Ć., Coffee, R. N., Cucini, R., Danailov, M., De Fanis, A., Demidovich, A., Ferrari, E., Finetti, P., Glaser, L., Knie, A., Lindahl, A. O., Plekan, O., Mahne, N., Mazza, T., Raimondi, L., Roussel, E., Scholz, F., Seltmann, J., Shevchuk, I., Svetina, C., Walter, P., Zangrando, M., Viefhaus, J., Grum-Grzhimailo, A. N. \& Meyer, M. (2018). Nature Communications, 9(1), 4659.

von Korff Schmising, C., Weder, D., Noll, T., Pfau, B., Hennecke, M., Strüber, C., Radu, I., Schneider, M., Staeck, S., Günther, C. M., Lüning, J., Merhe, A. e. d., Buck, J., Hartmann, G., Viefhaus, J., Treusch, R. \& Eisebitt, S. (2017). Review of Scientific Instruments, $\mathbf{8 8}(5), 053903$.

Kraus, P. M., Zürch, M., Cushing, S. K., Neumark, D. M. \& Leone, S. R. (2018). Nature Reviews Chemistry, 2(6), 82-94.

Lindle, D. W., Truesdale, C. M., Kobrin, P. H., Ferrett, T. A., Heimann, P. A., Becker, U., Kerkhoff, H. G. \& Shirley, D. A. (1984). The Journal of Chemical Physics, 81(12), $5375-5378$.

Lutman, A. A., MacArthur, J. P., Ilchen, M., Lindahl, A. O., Buck, J., Coffee, R. N., Dakovski, G. L., Dammann, L., Ding, Y., Dürr, H. A., Glaser, L., Grünert, J., Hartmann, G., Hartmann, N., Higley, D., Hirsch, K., Levashov, Y. I., Marinelli, A., Maxwell, T., Mitra, A., Moeller, S., Osipov, T., Peters, F., Planas, M., Shevchuk, I., Schlotter, W. F., Scholz, F., Seltmann, J., Viefhaus, J., Walter, P., Wolf, Z. R., Huang, Z. \& Nuhn, H. D. (2016). Nature Photonics, 10(7), 468-472.

Maltezopoulos, T., Laksman, J., Planas Carbonell, M., Dietrich, F., Liu, J., Freund, W., Koch, A., Jastrow, U. F., Sorokin, A., Tiedtke, K. \& Grünert, J. (2019). Journal of Synchrotron Radiation, This Volume.

Pellegrini, C., Marinelli, A. \& Reiche, S. (2016). Reviews of Modern Physics, 88(1), 1-55.

Prince, K., Vondráček, M., Karvonen, J., Coreno, M., Camilloni, R., Avaldi, L. \& de Simone, M. (1999). Journal of Electron Spectroscopy and Related Phenomena, 101-103, 141-147.

Roussel, E., Allaria, E., Callegari, C., Coreno, M., Cucini, R., Mitri, S., Diviacco, B., Ferrari, E., Finetti, P., Gauthier, D., Penco, G., Raimondi, L., Svetina, C., Zangrando, M., Beckmann, A., Glaser, L., Hartmann, G., Scholz, F., Seltmann, J., Shevchuk, I., Viefhaus, J. \& Giannessi, L. (2017). Photonics, 4(2), 29.

IUCr macros version 2.1.10: 2016/01/28 
Seddon, E. A., Clarke, J. A.., Dunning, D. J., Masciovecchio, C., Milne, C. J., Parmigiani, F., Rugg, D., Spence, J. C. H., Thompson, N. R., Ueda, K., Vinko, S. M., Wark, J. S. \& Wurth, W. (2017). Reports on Progress in Physics, 80(11), 115901.

Tiedtke, K., Azima, A., Von Bargen, N., Bittner, L., Bonfigt, S., Düsterer, S., Faatz, B., Frühling, U., Gensch, M., Gerth, C., Guerassimova, N., Hahn, U., Hans, T., Hesse, M., Honkavaar, K., Jastrow, U., Juranic, P., Kapitzki, S., Keitel, B., Kracht, T., Kuhlmann, M., Li, W. B., Martins, M., Núñez, T., Plönjes, E., Redlin, H., Saldin, E. L., Schneidmiller, E. A., Schneider, J. R., Schreiber, S., Stojanovic, N., Tavella, F., Toleikis, S., Treusch, R., Weigelt, H., Wellhöfer, M., Wabnitz, H., Yurkov, M. V. \& Feldhaus, J. (2009). New Journal of Physics, 11.

Tschentscher, T., Bressler, C., Grünert, J., Madsen, A., Mancuso, A. P., Meyer, M., Scherz, A., Sinn, H. \& Zastrau, U. (2017). Applied Sciences, 7(6).

Ullrich, J., Rudenko, A. \& Moshammer, R. (2012). Annual Review of Physical Chemistry, 63(1), 635-660.

Viefhaus, J., Scholz, F., Deinert, S., Glaser, L., Ilchen, M., Seltmann, J., Walter, P. \& Siewert, F. (2013). Nuclear Instruments and Methods in Physics Research Section A: Accelerators, Spectrometers, Detectors and Associated Equipment, 710, 151 - 154. The 4th international workshop on Metrology for X-ray Optics, Mirror Design, and Fabrication.

Yabashi, M., Tanaka, H., Tanaka, T., Tomizawa, H., Togashi, T., Nagasono, M., Ishikawa, T., Harries, J. R., Hikosaka, Y., Hishikawa, A., Nagaya, K., Saito, N., Shigemasa, E., Yamanouchi, K. \& Ueda, K. (2013). Journal of Physics B: Atomic, Molecular and Optical Physics, 46(16).

Yeh, J. \& Lindau, I. (1985). Atomic Data and Nuclear Data Tables, 32(1), 1-155.

Table 1. Photo emission lines for available gas targets.

\begin{tabular}{|c|c|c|c|c|}
\hline \multirow[t]{3}{*}{ Orbital } & \multicolumn{4}{|c|}{ Target gas } \\
\hline & $\mathbf{N}_{2}$ & $\mathrm{Ne}$ & $\mathbf{K r}$ & $\mathrm{Xe}$ \\
\hline & \multicolumn{4}{|c|}{ Photoelectron binding energy $[\mathrm{eV}]^{\dagger}$} \\
\hline K 1s & 409.9 & 870.2 & 14326 & 34561 \\
\hline $\mathrm{L}_{1} 2 \mathrm{~s}$ & $\overline{37.3}$ & 48.5 & 1921 & 5453 \\
\hline $\mathrm{L}_{2} 2 \mathrm{p}_{1 / 2}$ & 11.5 & 21.7 & 1730.9 & 5107 \\
\hline $\mathrm{L}_{3} 2 \mathrm{p}_{3 / 2}$ & 11.5 & 21.6 & 1678.4 & 4786 \\
\hline $\mathrm{M}_{1} 3 \mathrm{~s}$ & & & 292.8 & 1148.7 \\
\hline $\mathrm{M}_{2} 3 \mathrm{p}_{1 / 2}$ & & & 222.2 & 1002.1 \\
\hline $\mathrm{M}_{3} 3 \mathrm{p}_{3 / 2}$ & & & 214.4 & 940.6 \\
\hline $\mathrm{M}_{4} 3 \mathrm{~d}_{3 / 2}$ & & & 95.0 & 689.0 \\
\hline $\mathrm{M}_{5} 3 \mathrm{~d}_{5 / 2}$ & & & 93.8 & 676.4 \\
\hline $\mathrm{N}_{1} 4 \mathrm{~s}$ & & & 27.5 & 213.2 \\
\hline $\mathrm{N}_{2} 4 \mathrm{p}_{1 / 2}$ & & & 14.1 & 146.7 \\
\hline $\mathrm{N}_{3} 4 \mathrm{p}_{3 / 2}$ & & & 14.1 & 145.5 \\
\hline $\mathrm{N}_{4} 4 \mathrm{~d}_{3 / 2}$ & & & & 69.5 \\
\hline $\mathrm{N}_{5} 4 \mathrm{~d}_{5 / 2}$ & & & & 67.5 \\
\hline $\mathrm{O}_{1} 5 \mathrm{~s}$ & & & & 23.4 \\
\hline $\mathrm{O}_{2} 5 \mathrm{p}_{1 / 2}$ & & & & 13.4 \\
\hline $\mathrm{O}_{3} 5 \mathrm{p}_{3 / 2}$ & & & & 12.1 \\
\hline
\end{tabular}

${ }^{\dagger}$ Values taken from (Cardona \& Ley, 1978) 


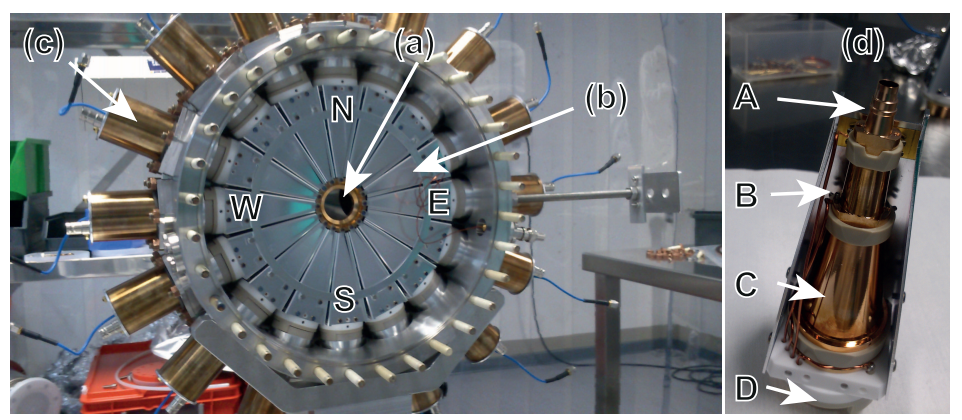

Fig. 1. Photoelectron Spectrometer assembled in the dedicated vacuum chamber. (a) In the interaction region, EuXFEL pulses ionize the effusive gas target which is injected via a capillary. (b) 16 electron TOF drifttubes provides polar angular resolution. (c) Each detector is mounted on a separate flange with feedthroughs for $\mathrm{HV}$ and signal readout. (d) Drifttube with shield partially disassembled to display the four segments [A B C D]. 


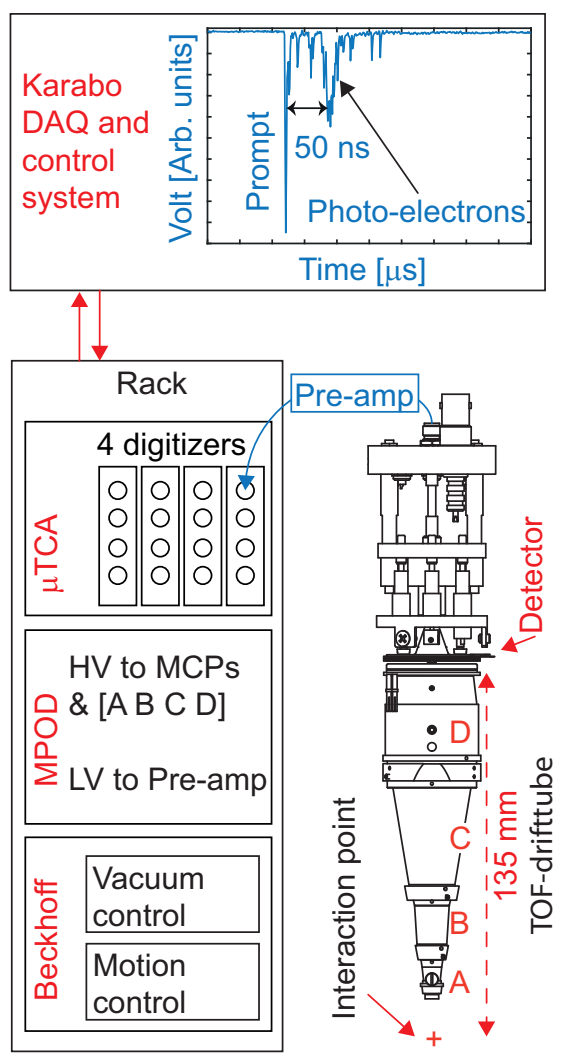

Fig. 2. Functional scheme of data acquisition and control system. Vacuum components and stepper motors are controlled via Beckhoff terminals. Voltages for drifttube segments [A B C D], Detectors, amplifiers and Helmholtz-coils are supplied via a MPOD crate. Detector signal is after amplification read by digitizers in a $\mu$ TCA crate. The presented plot is a typical single pulse spectrum where the first peak is the prompt from scattered light and the following peak is from photoelectrons. The rack containing all the electronics is remotely operated via the Karabo control system which is an environment designed for hardware control and data acquisition. 

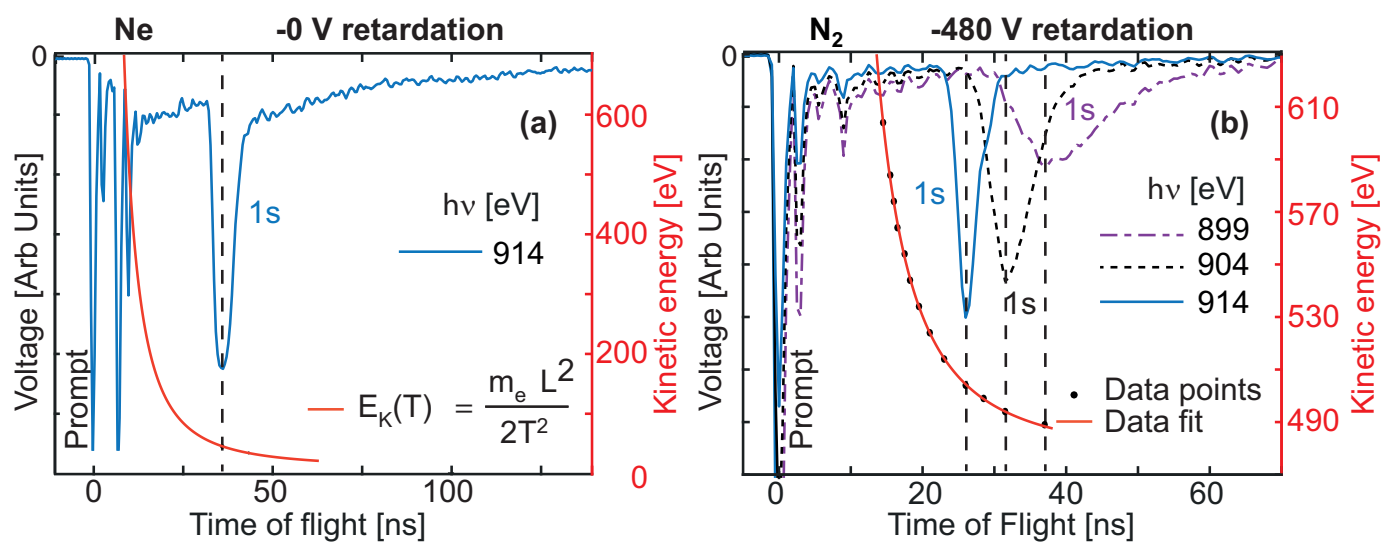

Fig. 3. (a) $0 \mathrm{~V}$ retardation. Electron TOF spectrum after Ne 1s ionization at $914 \mathrm{eV}$. TOF is $36.25 \mathrm{~ns}$ which corresponds to a kinetic energy of $44.2 \mathrm{eV}$ according to Eq. 1 (red curve). (b) $-480 \mathrm{~V}$ retardation. Electron TOF spectrum after $\mathrm{N}_{2}$ 1s ionization at different photon energies. Peak center (black dots) fitted to Eq. 2 (red curve). Spectra are averaged over 100 pulses for higher statistics. 


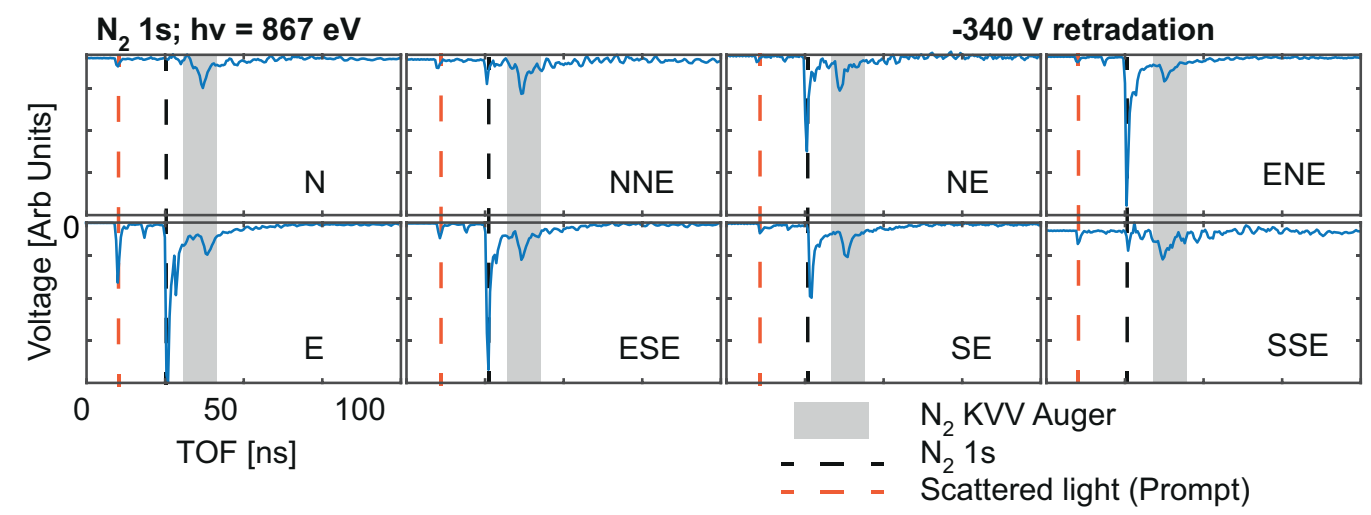

Fig. 4. Electron TOF spectrum after $\mathrm{N}_{2}$ ionization with $h \nu=867 \mathrm{eV}$ and pulse energy $\sim 234 \mu \mathrm{J}$. Retardation voltage is $-340 \mathrm{~V}$. In all spectra we detect $K V V$ peak with approximately constant intensity, which makes this peak ideal for detector performance calibration. $\mathrm{N}_{2} 1 \mathrm{~s}(\beta=2.00)$ on the other hand shows strong dependence on the polar axis. 


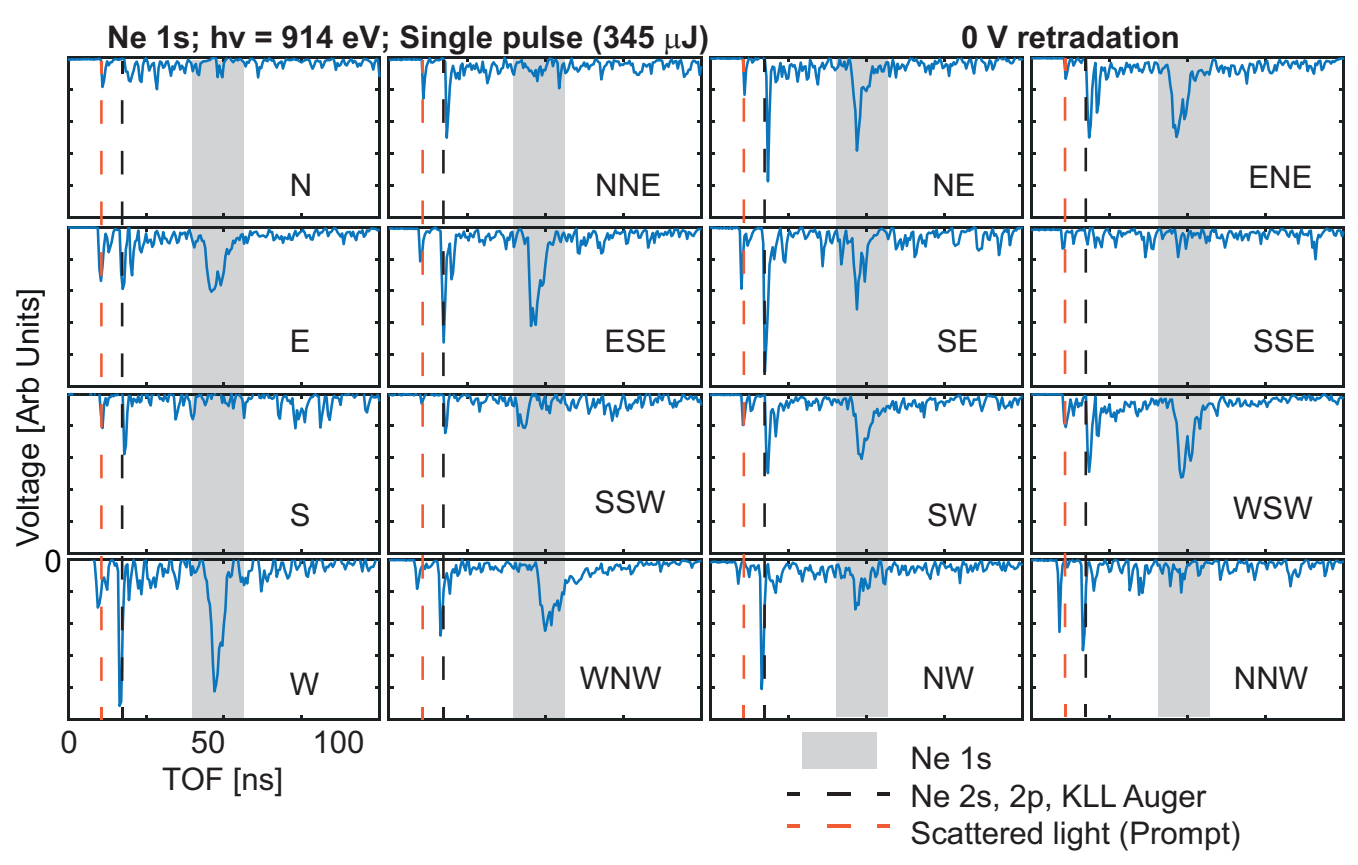

Fig. 5. TOF spectra for $\mathrm{Ne}$ at $h \nu=914 \mathrm{eV}$ with 16 electron TOF spectrometers oriented perpendicular with respect to X-ray beam. The spectra are the result of one single pulse from the SASE3 undulator. Red dashed lines indicate scattered light which defines the start time. Black dashed lines indicate the photoelectron lines $2 \mathrm{~s}$ and $2 \mathrm{p}$ and also $K L L$ Auger lines. Gray area indicates the 1 s peak. The Ne 1s photoelectron has $\beta=2.00$. The intensity distribution is dominating in the horizontal plane $E$ and $W$ and close to zero in the vertical direction $N$ and $S$ which indicates that the strongest component in the Stokes vector is the horizontal polarization. 

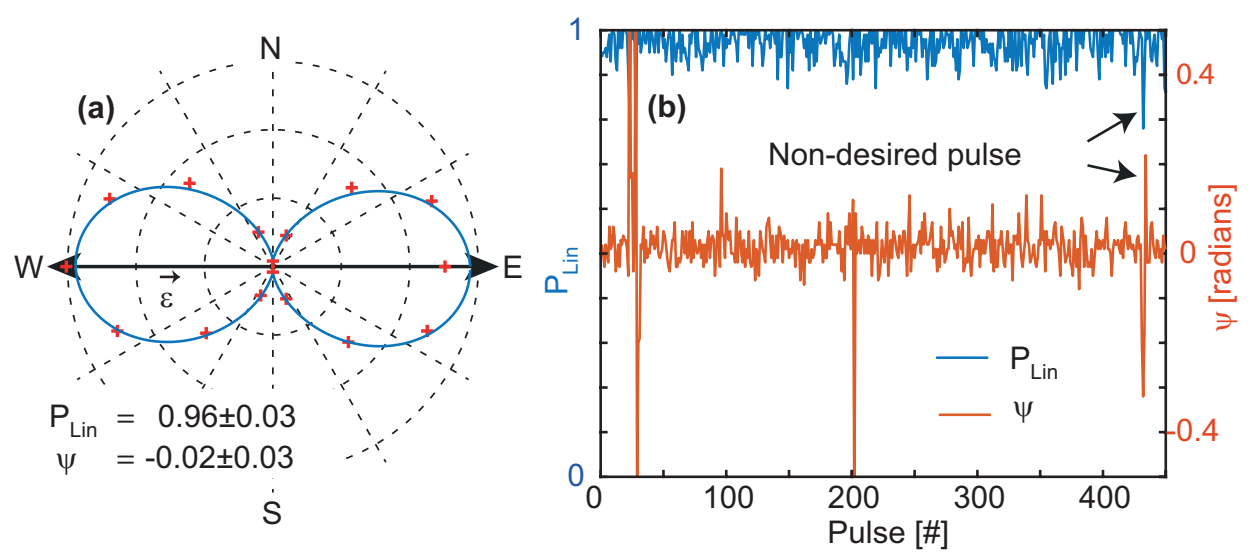

Fig. 6. Polariziation data of individual XFEL pulses collected with Ne 1 s at $h \nu=$ $914 \mathrm{eV}$. (a) Polar plot representation of intensity data from one pulse with fitted parameters $P_{\text {Lin }}$ and $\psi$ from Eq. 3. Arrow indicates polarization vector of light, $\vec{\varepsilon}$. (b) Polarization parameters for 450 consecutive pulses when machine is operated in single bunch mode. A few sharp spikes can be seen. Those are pulses with undesired properties.

\section{Synopsis}

The paper describes commissioning and first operation of an angle resolved Photoelectron Spectrometer for non-invasive shot-to-shot diagnostics for photon energy and polarization at the European XFEL soft X-ray beamline SASE3. 